TITLE:

\title{
Structure and phase transition of a uniaxially incommensurate In monolayer on $\mathrm{Si}(111)$
}

\section{$\operatorname{AUTHOR}(\mathrm{S}):$}

Terakawa, Shigemi; Hatta, Shinichiro; Okuyama, Hiroshi; Aruga, Tetsuya

\section{CITATION:}

Terakawa, Shigemi ... [et al]. Structure and phase transition of a uniaxially incommensurate In monolayer on Si(111). Physical Review B 2019, 100(11): 115428.

\section{ISSUE DATE:}

2019-09-15

URL:

http://hdl.handle.net/2433/244322

\section{RIGHT:}

(c) 2019 American Physical Society; 許諾条件に基づいて掲載しています $\circ$ 


\title{
Structure and phase transition of a uniaxially incommensurate In monolayer on $\mathrm{Si}(111)$
}

\author{
Shigemi Terakawa, Shinichiro Hatta $\odot$, Hiroshi Okuyama, and Tetsuya Aruga* \\ Department of Chemistry, Graduate School of Science, Kyoto University, Kyoto 606-8502, Japan
}

(Received 29 May 2019; revised manuscript received 28 August 2019; published 16 September 2019)

\begin{abstract}
We have studied the atomic structure and phase transition of a dense metallic monolayer of In on $\operatorname{Si}(111)$. Although the monolayer phase was previously considered to have a $(\sqrt{7} \times \sqrt{3})$ periodicity, low-energy electron diffraction and scanning tunneling microscopy observations have revealed that the phase has an incommensurate structure with the In overlayer uniaxially contracted by $2 \%$ from $(\sqrt{7} \times \sqrt{3})$. The uniaxially contracted structure was found to be more stable than the commensurate $(\sqrt{7} \times \sqrt{3})$ structure by first-principles calculations. We also observed a phase transition to a $(\sqrt{7} \times \sqrt{7})$ phase at 250-210 K upon cooling. Angle-resolved photoelectron spectroscopy and macroscopic four-point-probe conductivity measurements demonstrated that the transition induces the disappearance of metallic surface states and a sharp drop in sheet conductivity, respectively. These results indicate an electronic metal-insulator transition.
\end{abstract}

DOI: 10.1103/PhysRevB.100.115428

\section{INTRODUCTION}

Dense monolayers of $5 p$ and $6 p$ metals such as $\mathrm{In}, \mathrm{Tl}, \mathrm{Pb}$, and $\mathrm{Bi}$ on $\mathrm{Si}(111)$ or $\mathrm{Ge}(111)$ serve as intriguing systems to investigate physical properties of two-dimensional (2D) metals such as 2D superconductivity, Rashba spin splitting, and 2D electrical conduction [1-5]. These multivalent elements can both terminate the surface dangling bonds by $p_{z}$ orbitals and form 2D metallic bands with $p_{x}$ and $p_{y}$ orbitals $[4,6]$. The coverage often exceeds unity, which leads to a variety of ordered structures, including the ones incommensurate with the substrates $[7,8]$.

Metallic phases of $\operatorname{In} / \operatorname{Si}(111)$ have drawn considerable attention. The quasirectangular $(\sqrt{7} \times \sqrt{3})$ phase [hereafter $(\sqrt{7} \times \sqrt{3})$-rect] consisting of In double layers with fractional coverage $\theta=2.4$ [9-12] hosts 2D nearly-free-electron band structure [13] and exhibits a superconducting transition at $\sim 3 \mathrm{~K}[1,14,15]$. The $(4 \times 1)$ phase at $\theta=1.0$ has quasione-dimensional (quasi-1D) structure and undergoes a metalinsulator (MI) transition to an $(8 \times 2)$ phase at $\sim 120 \mathrm{~K}$ [16-18]. In addition, we recently revealed the metallic electronic structure of a dense monolayer phase, known as the quasihexagonal $(\sqrt{7} \times \sqrt{3})$ phase [hereafter $(\sqrt{7} \times \sqrt{3})$-hex] [19]. This phase is prepared in two different ways, one of which is high-temperature annealing of $\mathrm{In} / \mathrm{Si}(111)$ with a coverage of a little more than $\theta=1.0$ [20-22], and the other is deposition of In on the $\operatorname{In} / \operatorname{Si}(111)(\sqrt{3} \times \sqrt{3})$ surface at room temperature (RT) $[23,24]$. The former always results in the coexistence of the $(\sqrt{7} \times \sqrt{3})$-hex and $(4 \times 1)$ phases. Angleresolved photoelectron spectroscopy (ARPES) measurements have shown that the Fermi surface of the $(\sqrt{7} \times \sqrt{3})$-hex phase is consistent with that calculated theoretically for the monolayer model of $\theta=1.4$ [25].

The $(\sqrt{7} \times \sqrt{3})$-hex phase was reported to exhibit a structural transition to a $(\sqrt{7} \times \sqrt{7})$ phase upon cooling at $\sim 250 \mathrm{~K}$

*aruga@kuchem.kyoto-u.ac.jp
[23]. The electronic aspect of the phase transition has not been experimentally addressed. Recently, a question was raised about their coverages [24]. Since the $(\sqrt{7} \times \sqrt{3})$ and $(\sqrt{7} \times$ $\sqrt{7})$ unit cells contain mutually prime five and seven $(1 \times 1)$ unit cells, respectively, only integer values of coverage can be kept constant during the transition. This is not the case with $\theta=1.4$ of the $(\sqrt{7} \times \sqrt{3})$-hex phase.

In this work, we have investigated the atomic structure and phase transition of the $(\sqrt{7} \times \sqrt{3})$-hex phase with various surface analysis techniques, including low-energy electron diffraction (LEED), scanning tunneling microscopy (STM), four-point-probe (4PP) conductivity measurements, and ARPES. Analysis of LEED and STM observations revealed that the hex phase has an incommensurate structure that slightly deviates from $(\sqrt{7} \times \sqrt{3})$. The energetic stability of the incommensurate structure was examined by firstprinciples calculations. The coverage of the hex phase was found to be very close to $\theta=10 / 7$, the coverage of the $(\sqrt{7} \times$ $\sqrt{7})$ phase. We also found that the transition to the $(\sqrt{7} \times \sqrt{7})$ phase induces a rapid decrease in electrical conductivity and the disappearance of metallic surface states, indicating that the transition is associated with an electronic MI transition.

\section{EXPERIMENTAL AND COMPUTATIONAL METHODS}

All experiments were carried out in three different ultrahigh vacuum chambers. STM measurements (Unisoku USM1200) were performed at RT with an electrochemically etched tungsten tip in the constant-current mode. Surface electrical conductivity was measured in situ using a home-built 4PP with a probe spacing of $0.8 \mathrm{~mm}$. The 4PP apparatus was optimized for temperature-dependent measurement on semiconductor substrates over a range of 10-350 K [5] and was previously used for the observation of the MI transition of the $(4 \times 1)$ surface and hysteresis behavior [26]. Sheet resistivity $\rho(=1 / \sigma)$ was obtained by multiplying resistance $R$ (the slope of $I-V$ ) and a geometrical correction factor of 3.5 calculated from the layout of the contact points and the 


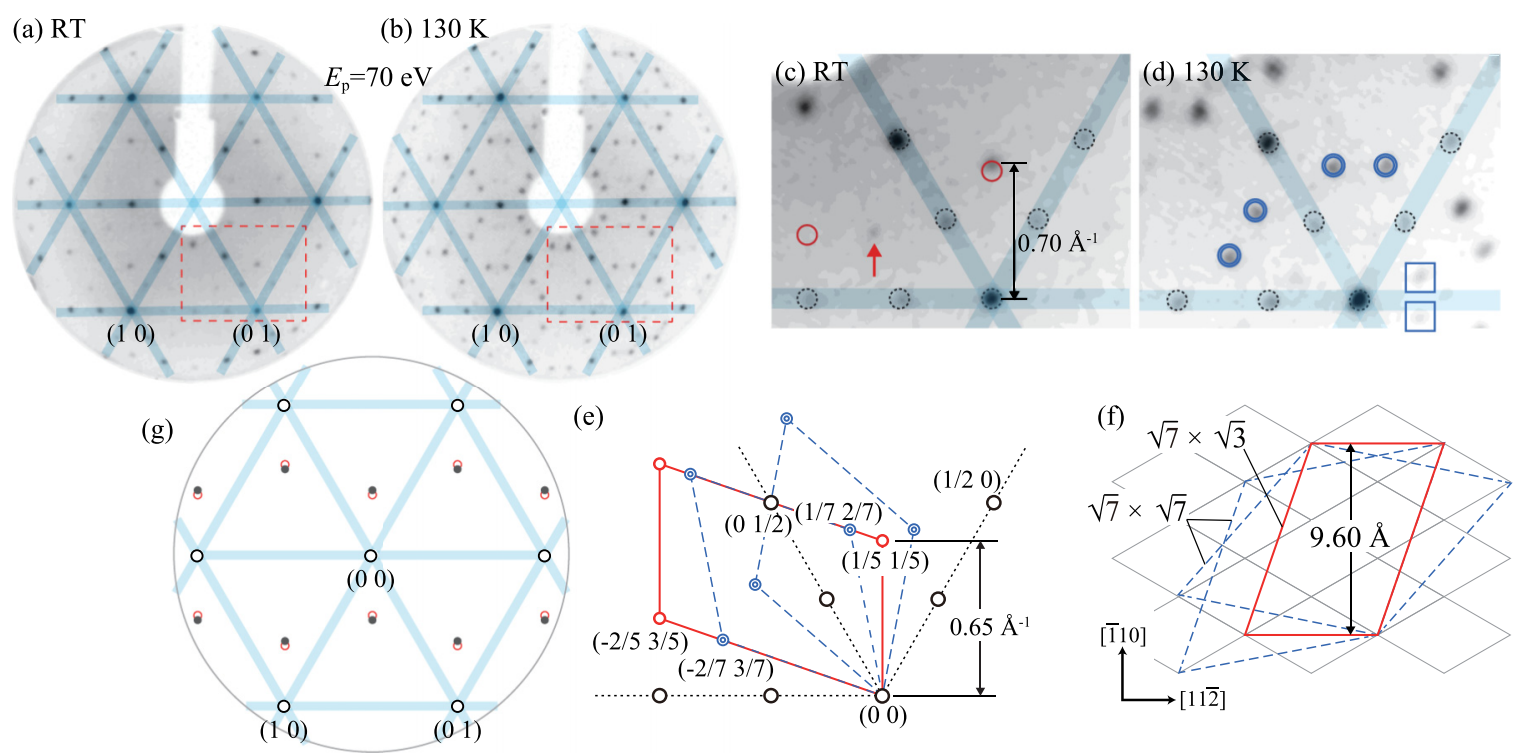

FIG. 1. LEED patterns of the surfaces with coexisting (a) $(\sqrt{7} \times \sqrt{3})$-hex and $(4 \times 1)$ phases at RT and (b) $(\sqrt{7} \times \sqrt{7})$ and $(4 \times 1)$ phases at $130 \mathrm{~K}$. The blue (gray) transparent lines indicate the $(1 \times 1)$ and $(4 \times 1)$ spots. $(\mathrm{c})$ and $(\mathrm{d})$ Enlarged images of the region indicated by the dashed rectangles in (a) and (b), respectively. The dotted black, solid red, and double blue circles represent the reciprocal lattice points of $(4 \times 1),(\sqrt{7} \times \sqrt{3})$, and $(\sqrt{7} \times \sqrt{7})$, respectively. The spot indicated by the arrow in (c) is the hex spot belonging to the minor domain rotated by $120^{\circ}$. Two weak spots enclosed by the squares in (d) are from the $(2 \sqrt{7} \times \sqrt{7})$ structure. (e) and (f) The reciprocal lattices of $(\sqrt{7} \times \sqrt{3})$ and $(\sqrt{7} \times \sqrt{7})$ and the corresponding real-space unit cells. The $(4 \times 1)$ reciprocal lattice points are also depicted in $(e)$. (g) Schematic of the spot pattern of the incommensurate hex phase. The neighboring $(\sqrt{7} \times \sqrt{3})$ spots are also depicted by the open red circles for easy identification of the positional relationship between the hex and $(1 \times 1)$ spots.

sample size [27]. Reproducibility was checked by measurements during repeated cycles of heating and cooling. ARPES experiments were done with a hemispherical electron energy analyzer (Scienta R3000) and $\mathrm{He} \mathrm{I} \alpha$ radiation $(h v=21.2 \mathrm{eV})$. $\mathrm{Si}(111)$ substrates were cleaned by direct-current heating at $1520 \mathrm{~K}$ for STM and $1320 \mathrm{~K}$ for ARPES and conductivity measurements. In the conductivity measurements, low-doped $n$-type $\operatorname{Si}(111)$ substrates $\left(\rho_{3 \mathrm{D}} \sim 1000 \Omega \mathrm{cm}\right)$ were used to sufficiently reduce bulk contribution. Highly doped $n$-type substrates $\left(\rho_{3 \mathrm{D}}<0.02 \Omega \mathrm{cm}\right)$ were used in the ARPES and STM measurements.

Total energy calculation was performed by applying the augmented plane waves plus local orbitals method implemented in the WIEN2K simulation package [28]. For the exchange-correlation potential the generalized gradient approximation was used. The In/Si(111) surfaces were modeled by repeated slabs of six layers of $\mathrm{Si}$ with one side covered with In atoms. The lattice constant $5.477 \AA$ of Si obtained by optimizing the bulk Si structure was used to construct the initial slab structure. The dangling bonds on the other side of the slab were terminated by $\mathrm{H}$ atoms. The structures were fully relaxed until the forces on all the atoms become smaller than $1 \mathrm{mRy} /$ bohr, while the bottommost $\mathrm{Si}$ and $\mathrm{H}$ atoms were fixed at the initial positions. $k$-point meshes of $3 \times 6 \times 1$ for $(\sqrt{7} \times \sqrt{3})$ unit cells and $2 \times 6 \times 1$ for $(7 \times \sqrt{3})$ unit cells were used.

\section{RESULTS AND DISCUSSION}

\section{A. Structure analysis by LEED and STM}

The In/Si(111) $(\sqrt{7} \times \sqrt{3})$-hex phase was prepared by deposition of $\operatorname{In}(\theta=1.2)$ on the $\operatorname{Si}(111)(7 \times 7)$ surface at RT, followed by annealing at $640 \mathrm{~K}$ for several tens of seconds. This gave $(\sqrt{7} \times \sqrt{3})$-hex domains coexisting with domains of $(4 \times 1)(\theta=1.0)$ with nearly equal area ratios [19] [see Fig. 2(a) below]. Figure 1(a) shows the LEED pattern of the surface at RT. The triple-domain $(4 \times 1)$ spots are aligned along the lines passing through the fundamental $(1 \times 1)$ spots, as indicated by the blue (gray) lines. The triple-domain hex spots are also seen, but one domain is always dominant, as reported in our previous study [19].

We could not increase the area of the $(\sqrt{7} \times \sqrt{3})$-hex phase by increasing the initial In coverage, which instead resulted in the formation of the double-layer $(\sqrt{7} \times \sqrt{3})$-rect phase $(\theta=2.4)$ coexisting with the hex and $(4 \times 1)$ phases. Annealing at $640 \mathrm{~K}$ was essential to obtain well-ordered hex phase domains. However, prolonged annealing decreased the hex phase due to desorption of In and made the $(4 \times 1)$ phase dominant. On the other hand, the hex phase was not reproduced by the In deposition onto the $(4 \times 1)$ phase followed by annealing. These results suggest that the $(\sqrt{7} \times \sqrt{3})$-hex structure is metastable and the thermally induced change to $(4 \times 1)$ is irreversible.

Upon cooling from RT, the LEED pattern changed to $(\sqrt{7} \times \sqrt{7})+(4 \times 1)[$ Fig. $1(\mathrm{~b}), 130 \mathrm{~K}]$. The $(\sqrt{7} \times \sqrt{7})$ pattern shows coexistence of two domains which are symmetric with respect to the mirror planes of $\mathrm{Si}(111)$. The transition was also observed on the surface prepared by RT deposition of In on the $(\sqrt{3} \times \sqrt{3})$ surface (not shown). However, the transition was rather diffuse; the weak LEED spots of the hex phase were observed even at $100 \mathrm{~K}$. Similar results were reported in previous LEED observations [12,24]. The broadening of the transition is most likely due to the insufficient ordering of the hex surface. Consequently, the coexisting surface prepared by 
the first method is found to be appropriate for studying the phase transition. The details of the change in LEED patterns as a function of temperature will be described later.

Figures 1(c) and 1(d) show close-up images of the LEED patterns of Figs. 1(a) and 1(b), respectively. We determined the coordinates of reciprocal lattice points of single-domain $(\sqrt{7} \times \sqrt{3})$ and double-domain $(\sqrt{7} \times \sqrt{7})$ with respect to the positions of the $(1 \times 1)$ and $(4 \times 1)$ spots, which are shown by open circles in Figs. 1(c) and 1(d). Corresponding reciprocal and real lattices of $(\sqrt{7} \times \sqrt{3})$ and $(\sqrt{7} \times \sqrt{7})$ are depicted in Figs. 1(e) and 1(f), respectively. While good agreement between the LEED spots and the reciprocal lattice points is seen for the $(\sqrt{7} \times \sqrt{7})$ phase [Fig. 1(d)], the LEED spot of the hex phase deviates from the $(\sqrt{7} \times \sqrt{3})$ lattice point [Fig. 1(c)]. The deviation of the spot positions in the hex phase was also reported in a recent LEED observation [12]. A schematic of the overall spot pattern of the hex phase is shown in Fig. 1(g) along with that of $(\sqrt{7} \times \sqrt{3})$.

The hex spots appear as the satellite spots around each $(1 \times 1)$ spot with the constant distance indicated by the double-headed arrow in Fig. 1(c). These spots are interpreted to result from the double scattering by the $\mathrm{Si}(111)$ substrate and the incommensurate overlayer. We measured the distance for all equivalent pairs of spots in Fig. 1(a). This gives $8.92 \pm 0.10 \AA$ for the separation between lattice lines parallel to $[11 \overline{2}]$, which is $93 \%$ of that for the $(\sqrt{7} \times \sqrt{3})$ lattice lines [9.60 A, Fig. 1(f)]. On the other hand, we were not able to examine the other group of lattice lines due to the absence of spots corresponding to $(-2 / 53 / 5)$ irrespective of electron energy [Figs. 1(c) and 1(e)].

Figure 2(a) shows a large-scale STM image of the twophase coexisting surface at RT. The brighter areas on a terrace correspond to the hex phase, and the darker ones correspond to the $(4 \times 1)$ phase. Both of the phases have large domain sizes with nearly equal distribution on a terrace. A close-up image of a region including the phase boundary is shown in Fig. 2(b). The 1D chains with narrower spacing along [11 $\overline{2}]$ in the bottom right part correspond to the hex phase. The distance between the chains was determined to be $8.8 \pm 0.5 \AA$ with reference to that of the $(4 \times 1)$ phase. This distance is consistent with that obtained from the above LEED analysis. Atomically resolved STM images of the hex phase were observed under relatively low bias conditions $\left(V_{\mathrm{S}}<+0.8 \mathrm{~V}\right)$. In Fig. 2(c), atomic chains are clearly seen together with boundaries between domains rotated by $120^{\circ}$. A closer look shows that the chains are classified into two kinds of structure: the "zigzag" type and the "linear" type, each of which is enlarged in Fig. 2(d). Their periodicities along the chains are well defined and coincide with each other. The period was evaluated to be $\sqrt{3}$ times as long as the surface lattice constant of $\operatorname{Si}(111)(1 \times 1)$. On the other hand, the two types of chains appear in an irregular order with a nearly equal frequency, as indicated by the letters $\mathrm{Z}$ and $\mathrm{L}$ in Fig. 2(c). Note also that the spatial phase among chains along the chain direction is not ordered. These should be the reasons why the LEED spots corresponding to $(-2 / 53 / 5)$ for $(\sqrt{7} \times \sqrt{3})$ were not observed.

From the above analysis, the incommensurability of the In overlayer with respect to the substrate lattice is revealed along the [110] direction, which is perpendicular to the chains. Thus,
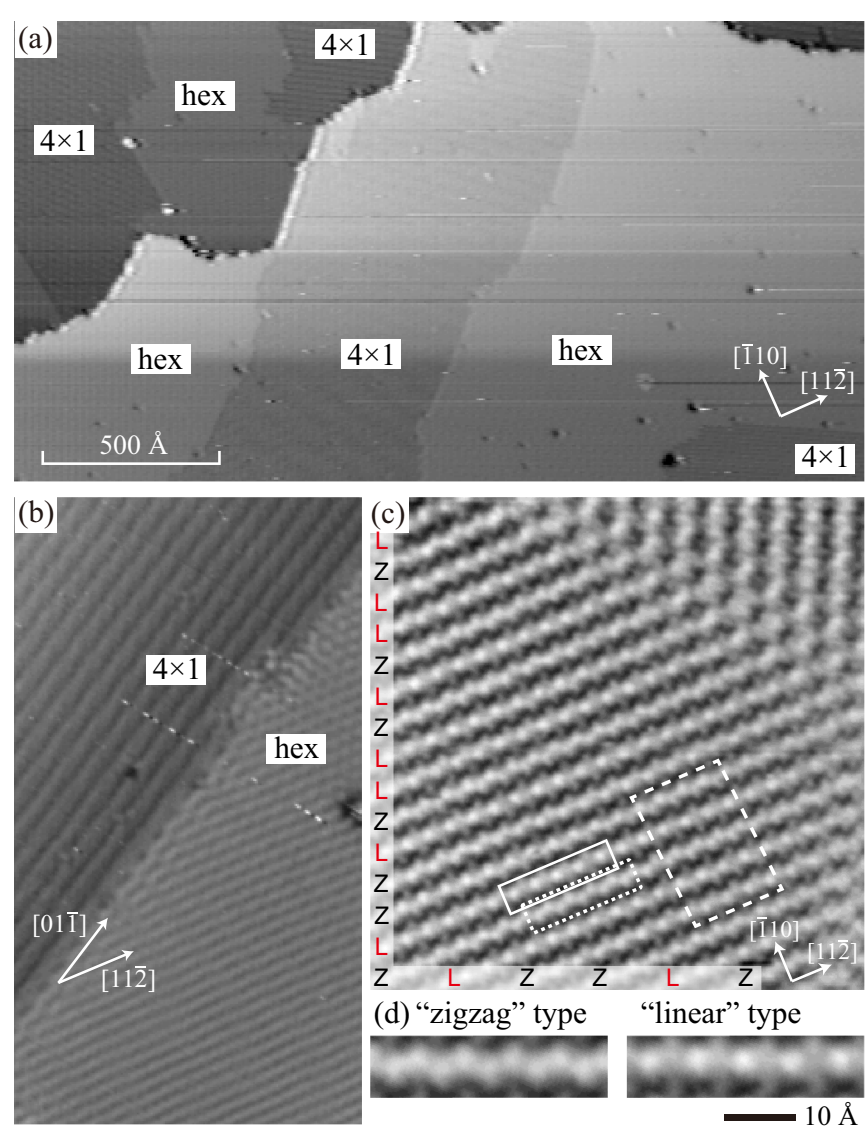

FIG. 2. (a) Large-scale STM image $\left(2400 \times 1300 \AA^{2}\right)$ of the surface covered with the hex and $(4 \times 1)$ phases, acquired at sample bias $V_{\mathrm{s}}=-2.0 \mathrm{~V}$ and tunneling current $I=0.1 \mathrm{nA}$. (b) STM image $\left(220 \times 400 \AA^{2}\right)$ of the adjacent hex and $(4 \times 1)$ domains $\left(V_{\mathrm{s}}=+1.5 \mathrm{~V}, I=0.1 \mathrm{nA}\right)$. (c) High-resolution STM image $(140 \times$ $\left.140 \AA^{2}\right)$ of the hex phase $\left(V_{\mathrm{s}}=+0.3 \mathrm{~V}, I=0.1 \mathrm{nA}\right)$. (d) Enlarged images of the (left) zigzag and (right) linear-type atomic chains of the hex phase, clipped from the dotted and solid rectangles in (c), respectively. The letters $\mathrm{Z}$ and $\mathrm{L}$ on the left and bottom sides of (c) indicate the types of the chains. The area enclosed by the dashed box corresponds to the structure model shown in Fig. 3(b). All the STM images were taken at RT.

we withdraw the term " $(\sqrt{7} \times \sqrt{3})$ " from the name of this phase hereafter. STM demonstrated that the chains of atomic protrusions are the key feature of the pseudoperiod of $8.9 \AA$ observed by LEED.

Let us now consider the atomic structure of the incommensurate overlayer on the basis of the chain structure along [112] . A zigzag chain structure is found in the $\theta=1.4$ model [25], for which calculated Fermi surface was supported by ARPES [19]. According to the $\theta=1.4$ model, In atoms are located away from the $T_{4}$ sites. The seven In atoms in the $(\sqrt{7} \times \sqrt{3})$ unit cell are uniformly distributed along the [110] direction, as indicated by the dashed lines on the left side of Fig. 3(a). Accordingly, the structure can be regarded as consisting of In rows running along [11르. The average interrow distance $\lambda_{\operatorname{In}}$ is $1.37 \AA$ in the $\theta=1.4$ model. The chain structure consists of rows of In atoms located near the 
(a)

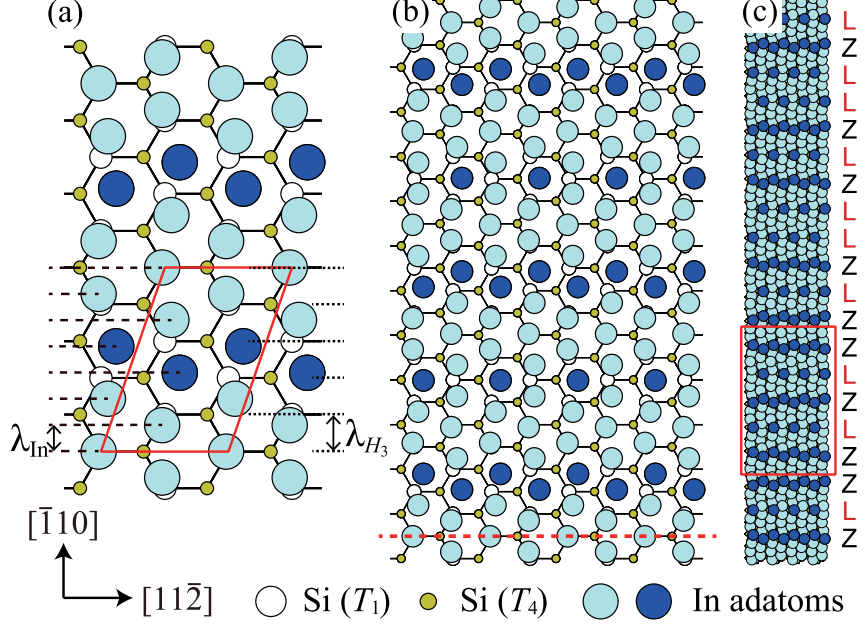

FIG. 3. (a) Schematic of the $\theta=1.4$ model with the $(\sqrt{7} \times \sqrt{3})$ periodicity given by Park and Kang [25]. The parallelogram indicates the $(\sqrt{7} \times \sqrt{3})$ unit cell. (b) and (c) The model of the incommensurate hex phase. The darkly colored In adatoms are close to the $\mathrm{H}_{3}$ sites and protrude more than the brightly colored ones. The rectangle in (c) marks the region enlarged in (b). The letters $\mathrm{Z}$ and $\mathrm{L}$ on the right side indicate the types of the atomic chains.

$H_{3}$ sites of $\mathrm{Si}(111)$ that protrude due to the weak interaction with the substrate Si atoms [Fig. 3(a)].

Now we assume a weak contraction of the In overlayer along the [110] direction. This causes the shift of the position of each In row along [110], which leads to the modulation of the period of protruding In atom rows. In order to evaluate the degree of contraction, we used a model of superposition of two 1D waves with different wave vectors $k_{i}=2 \pi / \lambda_{i}(i=$ In, $H_{3}$ ). The period of the $H_{3}$ sites along [ $\left.\overline{1} 10\right]$ is half of the surface lattice constant of $\operatorname{Si}(111)(1 \times 1)$ [the dotted lines on the right side of Fig. 3(a)], and thus, $k_{H_{3}}=3.27 \AA^{-1}$. The superposed wave has wave vectors $k_{ \pm}=\left(k_{\mathrm{In}} \pm k_{H_{3}}\right) / 2$. Since $k_{-}$is appropriate for the period of protruding atoms $\left(\lambda_{-}=\right.$ $\left.2 \pi / k_{-}=8.92 \AA\right)$, we obtain $k_{\text {In }}=4.68 \AA^{-1}$ and hence $\lambda_{\text {In }}=$ $1.34 \AA$, which is $2.1 \% \pm 0.3 \%$ smaller than the average interrow distance of the $\theta=1.4$ model.

Figures 3(b) and 3(c) show a structure model of the hex phase constructed as follows. First, the In atoms on the dashed line in Fig. 3(b) are placed at the $T_{1}$ sites, and then, other In rows along [11ㄹ] are arranged with an equal distance of $1.34 \AA$, while the atom positions in each row are adjusted to be consistent with the local geometry in the $\theta=1.4$ model. As a result, linear and zigzag chain structures appear as indicated by the dark blue (gray) circles in Figs. 3(b) and 3(c). The former is composed of single rows of In atoms almost on the $\mathrm{H}_{3}$ sites, whereas the latter is composed of paired rows of In atoms slightly shifted from the $H_{3}$ sites. The arrangement of the two types of chains is sensitive to the degree of the contraction. We found that the model shows good agreement with the observed STM image at the contraction of 2.4\%; the character string L-Z-L-L-... in Fig. 3(c) perfectly matches that in Fig. 2(c). The obtained contraction is a little larger than that derived from the LEED analysis, which may suggest the

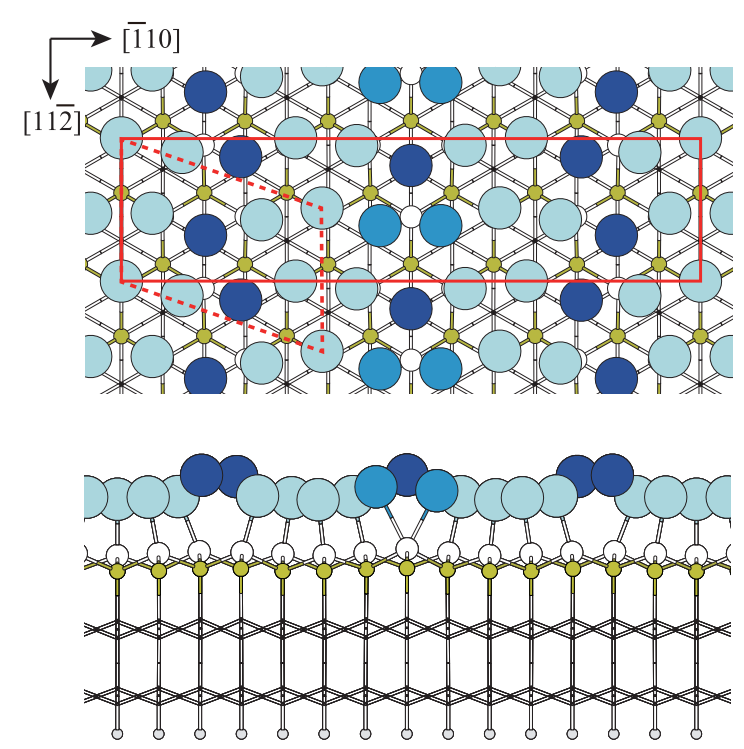

FIG. 4. Top and side views of the optimized structure of the $(7 \times \sqrt{3})$ slab model. The rectangle indicates the $(7 \times \sqrt{3})$ unit cell. The dashed line indicates the unit cell of the $\theta=1.4$ model, which, however, is contracted uniaxially by $2 \%$ along [ $[\overline{1} 10]$.

spatial fluctuation of the incommensurate structure depending on local conditions such as domain sizes and boundaries.

\section{B. First-principles total-energy calculation}

In order to examine whether the contraction of the In overlayer is energetically favorable, we performed a firstprinciples total energy calculation for the $(\sqrt{7} \times \sqrt{3})$ model $[\theta=7 / 5(1.4)]$ [25] and a modified model in which the In overlayer is contracted along [1110]. We adopted a long-period commensurate structure $(7 \times \sqrt{3})$ with $\theta=20 / 14=1.43$ as a model of the incommensurate In overlayer. Starting from approximate atomic positions similar to that shown in Fig. 3(b), the atomic positions were extensively relaxed to obtain the total energies, which converged well within $1 \mathrm{meV}$.

The optimized structure is shown in Fig. 4. The Z-L-Z chain sequence, which is frequently observed in the STM image [Fig. 2(c)], is reproduced. In the STM image, the two $\mathrm{Z}$ chains in the Z-L-Z triple are always in antiphase, and the phase relation of $L$ to $Z$ is always fixed. These characteristics are precisely reproduced in the calculated structure.

The mass balance associated with the contraction of the $(\sqrt{7} \times \sqrt{3})$ structure to $(7 \times \sqrt{3})$ is represented as

$$
\frac{2}{5} \operatorname{In}(\mathrm{ref})+\frac{14}{5} \operatorname{In}_{7}\left(\mathrm{Si}_{6} \mathrm{H}\right)_{5} \rightarrow \operatorname{In}_{20}\left(\mathrm{Si}_{6} \mathrm{H}\right)_{14},
$$

where $\mathrm{Si}_{6} \mathrm{H}$ indicates the $(1 \times 1)$ unit cell of the substrate slab. $\operatorname{In}_{7}\left(\mathrm{Si}_{6} \mathrm{H}\right)_{5}$ and $\operatorname{In}_{20}\left(\mathrm{Si}_{6} \mathrm{H}\right)_{14}$ denote the slab models for $(\sqrt{7} \times \sqrt{3})(\theta=1.4)$ and $(7 \times \sqrt{3})(\theta=1.43)$, respectively. $\mathrm{In}(\mathrm{ref})$ denotes the In atom which reacts with the $(\sqrt{7} \times \sqrt{3})$ structure to form the contracted $(7 \times \sqrt{3})$ structure.

The energy change associated with the contraction per $(\sqrt{7} \times \sqrt{3})$ unit cell $\Delta E_{\mathrm{c}}$ is given as

$$
\Delta E_{\mathrm{c}}=\frac{5}{14}\left[E_{7 \times \sqrt{3}}-\left(0.4 E_{\mathrm{In}}+2.8 E_{\sqrt{7} \times \sqrt{3}}\right)\right],
$$


where $E_{7 \times \sqrt{3}}$ and $E_{\sqrt{7} \times \sqrt{3}}$ denote the total energies of the optimized $(7 \times \sqrt{3})$ and $(\sqrt{7} \times \sqrt{3})$ structures, respectively. $E_{\text {In }}$ is the energy per atom of In that is to be incorporated into the $\sqrt{7} \times \sqrt{3}$ structure and causes the contraction.

In order to examine the relative stability of the contracted structure to the uncontracted $(\sqrt{7} \times \sqrt{3})$ structure, the reference state of indium, In(ref), should be specified. In atoms in the $(4 \times 1)$ structure coexisting with the $(\sqrt{7} \times \sqrt{3})$ domains are not appropriate because, as already discussed, the $(4 \times 1)$ structure is highly stable and the transfer of In atoms from the $(4 \times 1)$ to $(\sqrt{7} \times \sqrt{3})$ phase is implausible. Another candidate is an In atom in bulk In islands, which would be formed during the initial In deposition on the clean $\mathrm{Si}(111)$ surface. However, the wide-area STM image of the annealed surface in Fig. 2(a) does not show In islands remaining on the surface, which suggests that In islands are not in equilibrium with the contracted hex structure at least at a later stage of the formation of the ordered structures.

A plausible candidate is an isolated In adatom, which should exist throughout the formation process of the surface covered with the hex and $(4 \times 1)$ structures. In that case, the stability of the contracted structure depends on the energetics of the incorporation of In adatoms into the $(\sqrt{7} \times \sqrt{3})$ structure.

In order to determine $\operatorname{In}($ ref $)$ and $E_{\mathrm{In}}$, the energy of an In adatom on the $\theta=1.4(\sqrt{7} \times \sqrt{3})$ structure was calculated for two different adsorption sites: a threefold hollow site over In atoms in the "trough" between protruding In rows (trough site) and a site at which the In adatom is bonded with two protruding In atoms and a trough atom (protrusion site). The energy of the In adatom was obtained by fully relaxing the positions of the In adatom as well as the substrate atoms. We found that the In adatom adsorbed on the trough site is more stable by $88 \mathrm{meV}$. The energy per atom of In adsorbed on the trough site was found to be even lower than that in solid In because of the relaxation of the $(\sqrt{7} \times \sqrt{3})$ In overlayer. Note that the In adatom in this case was never incorporated into the In layer but located at $2.45 \AA$ above the threefold hollow site of the In layer because it would result in a too dense $(\theta=1.6)$ In overlayer. We chose as $\operatorname{In}(\mathrm{ref})$ the In adatom on the trough site of the uncontracted $(\sqrt{7} \times \sqrt{3})$ surface.

The contraction energy $\Delta E_{\mathrm{c}}$ of the contracted model $(\theta=1.43)$ was obtained from Eq. (1) to be $-18 \mathrm{meV}$ per $(\sqrt{7} \times \sqrt{3})$ unit cell. The result suggests that the $(\sqrt{7} \times \sqrt{3})$ structure with In adatoms is not energetically stable and will be transformed to the contracted structure by accepting In adatoms into the monolayer itself. This result is consistent with the experimental observation of the contracted In monolayer of $\theta=1.43$.

\section{Phase transitions}

In a previous report [19], we presented the Fermi surface measured for the hex phase, which was composed of circles centered at the $(1 \times 1) \bar{\Gamma}$ points. Considering the incommensurate structure of the hex phase, no observation of the periodic behavior according to the $(\sqrt{7} \times \sqrt{3})$ surface Brillouin zone (SBZ) was reasonable. While we compared the Fermi surface folded into the $(\sqrt{7} \times \sqrt{3}) \mathrm{SBZ}$ with that calculated
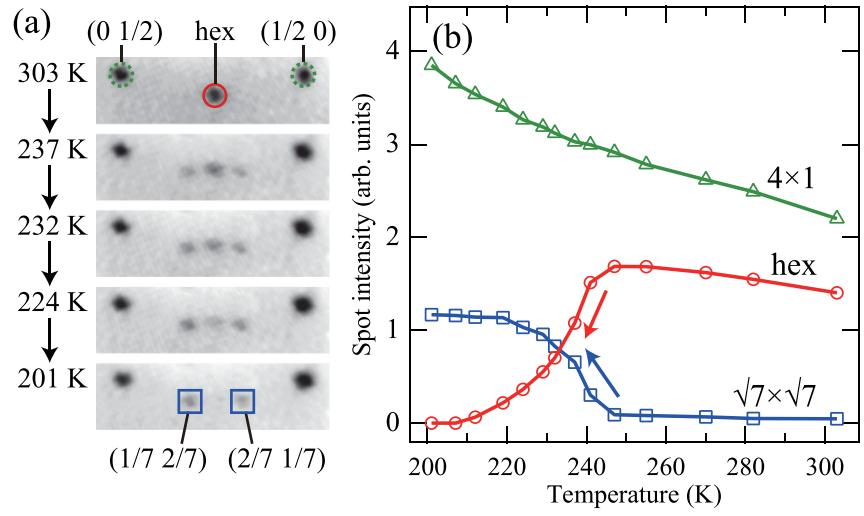

FIG. 5. (a) Close-up views of the LEED patterns measured during cooling $\left(E_{\mathrm{p}}=56 \mathrm{eV}\right)$. The solid red circle, blue squares, and dotted green circles indicate the spots of the hex, $(\sqrt{7} \times \sqrt{7})$, and $(4 \times 1)$ phases, respectively. (b) The intensities of the superstructure spots of the three phases as a function of temperature upon cooling.

for the $\theta=1.4$ model [Figs. 4(a) and 4(b) in Ref. [19]], it is now clear that it was not appropriate to examine the details of the Fermi contours. Instead, it is meaningful to compare the experimental and theoretical Fermi surfaces in the extended zone scheme. The Fermi wave vector of the hex phase along the direction perpendicular to the contraction is still very consistent with that of the $\theta=1.4$ model.

The contracted hex structure has an In coverage of 1.43. This is very close to $\theta=10 / 7$ of the $(\sqrt{7} \times \sqrt{7})$ model, which contains ten In atoms per unit cell [25], suggesting that the transfer of In with neighboring phases should be negligible during the transition between the hex and $(\sqrt{7} \times \sqrt{7})$ phases.

Figure 5(a) shows the LEED pattern change during cooling the surface with coexisting hex and $(4 \times 1)$ phases from RT to $200 \mathrm{~K}$. The half-order spots are due to the $(4 \times 1)$ phase. The $(\sqrt{7} \times \sqrt{7})$ spots are seen below $240 \mathrm{~K}$ on both sides of the hex spot. When the temperature reaches $200 \mathrm{~K}$, the hex spot completely disappears. At intermediate temperatures, the hex-phase and $(\sqrt{7} \times \sqrt{7})$ spots are simultaneously observed. Figure 5(b) shows the integrated intensities of the spots as a function of temperature. With decreasing temperature, the spot intensity of the hex phase decreases at $250-210 \mathrm{~K}$, and that of $(\sqrt{7} \times \sqrt{7})$ increases concomitantly, while that of $(4 \times$ 1) increases monotonically according to the Debye-Waller effect. As to the spot widths and positions, temperaturedependent change is negligible for all the spots.

Figure 6(a) shows the temperature dependence of the sheet conductivity of the surface with coexisting hex and $(4 \times 1)$ phases during cooling from RT to $67 \mathrm{~K}$ and subsequent heating. The rate of temperature increase and decrease was $\pm 2.4 \mathrm{~K} / \mathrm{min}$. Upon cooling from RT, $\sigma$ shows a sharp drop at $T_{1 \downarrow}=250 \mathrm{~K}$. The rapid decrease in $\sigma$ declines at $\sim 210 \mathrm{~K}$. The change of $\sigma$ at 250-210 K corresponds to the LEED pattern change from hex to $(\sqrt{7} \times \sqrt{7})$ observed at this temperature range. After the gradual change, $\sigma$ shows a drop again at $T_{2 \downarrow}=118 \mathrm{~K}$. The second drop is attributed to the MI transition from $(4 \times 1)$ to $(8 \times 2)$. Upon heating, $\sigma$ increases with temperature, while the increase shows rapid deceleration twice, at $T_{2 \uparrow}=128 \mathrm{~K}$ and $T_{1 \uparrow}=291 \mathrm{~K}$. The overall behavior of $\sigma$ indicates that both the transitions show hystereses, 

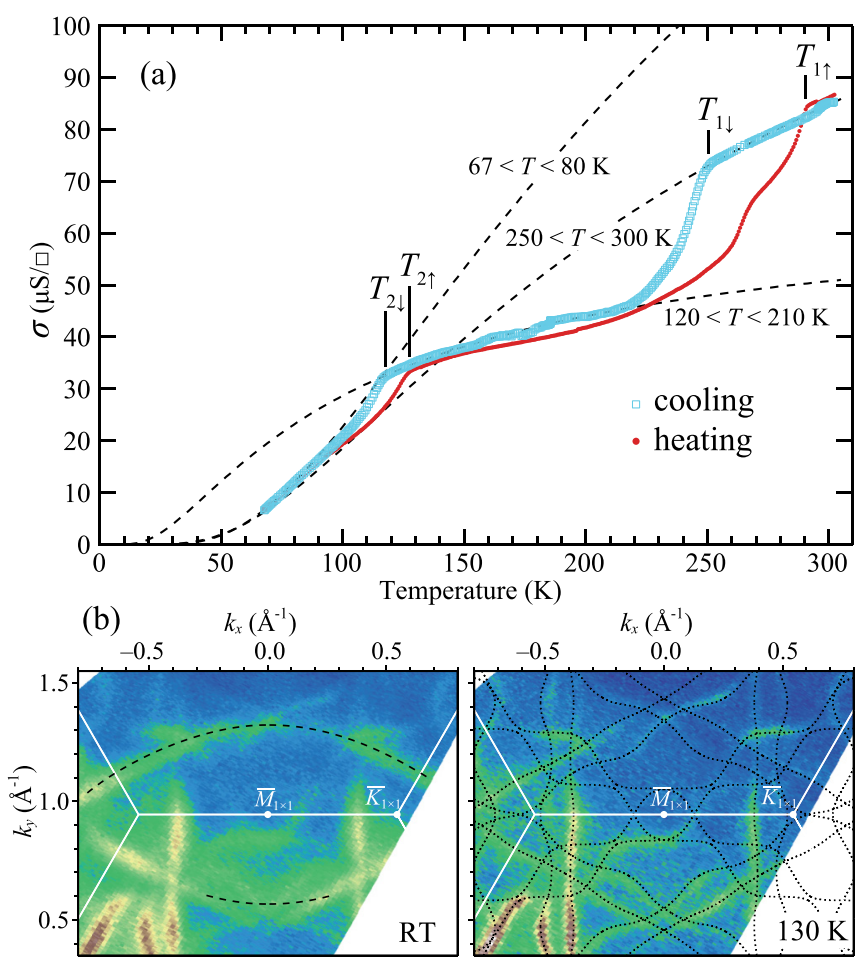

FIG. 6. (a) Temperature dependence of $\sigma$ of the surface with coexisting hex and $(4 \times 1)$ phases measured during cooling [blue (light gray)] and heating [red (dark gray)]. Fitting curves are also shown as dashed lines. (b) Fermi surface maps of the surfaces with coexisting (left) hex and $(4 \times 1)$ phases at RT and (right) $(\sqrt{7} \times \sqrt{7})$ and $(4 \times 1)$ phases at $130 \mathrm{~K}$. The maps were obtained with an energy window of $50 \mathrm{meV}$. The solid lines represent the $(1 \times 1)$ SBZs. The dashed curves indicate the observed Fermi surface of the hex phase. The dotted curves represent the replica of the Fermi surfaces of the triple-domain $(4 \times 1)$ phase $[17,18]$.

suggesting that the transitions are of first order [26]. The observed hysteresis width for the $(4 \times 1)-(8 \times 2)$ transition is $10 \mathrm{~K}$, and that for the hex $-(\sqrt{7} \times \sqrt{7})$ transition is $41 \mathrm{~K}$.

As to the $(\sqrt{7} \times \sqrt{7})$ phase, further transitions on cooling below $200 \mathrm{~K}$ were reported in a previous STM study [23]; several long-period superstructures, $p \sqrt{7} \times \sqrt{7}(p=2,3,6)$ and $\frac{10}{3} \sqrt{3} \times \frac{40}{3} \sqrt{3}$, were observed. Among them, the $(2 \sqrt{7} \times$ $\sqrt{7}$ ) superstructure was also observed in our LEED measurement at 82-200 K [see Fig. 1(d)]. However, the intensity of the $(2 \sqrt{7} \times \sqrt{7})$ spots remained much weaker than that of the $(\sqrt{7} \times \sqrt{7})$ spots, which indicates that the transition to $(2 \sqrt{7} \times \sqrt{7})$ takes place only at a very limited part of the $(\sqrt{7} \times \sqrt{7})$ domains, implying that long-period structures such as $(2 \sqrt{7} \times \sqrt{7})$ are defect-induced local structures, as suggested in a previous study [23].

As shown in the STM images in Fig. 2, the preparation of the hex phase by high-temperature annealing provides welldeveloped hex-phase domains with a low density of defects. The good ordering of the hex phase results in the transition to the well-developed $(\sqrt{7} \times \sqrt{7})$ phase with negligible defectinduced structures. By taking care of these points, we obtained the well-ordered $(\sqrt{7} \times \sqrt{7})$ and $(4 \times 1)$ phases between 210 and $\sim 120 \mathrm{~K}$ and the $(\sqrt{7} \times \sqrt{7})$ and $(8 \times 2)$ phases below $\sim 80 \mathrm{~K}$. In the intermediate-temperature range, $80-130 \mathrm{~K}$, the first-order MI transition between the $(4 \times 1)$ and $(8 \times 2)$ phases takes place.

The $\sigma-T$ curves exhibit nonmetallic temperature dependence in the entire temperature range, while the hex and $(4 \times 1)$ phases have metallic band structures. Similar behavior was also observed in the $(4 \times 1)$ phase and was suggested to be indicative of hopping conduction between metallic domains separated by boundaries between the $(4 \times 1)$ domains rotated by $120^{\circ}$, acting as line defects [26]. As shown in Fig. 6(a), the data during cooling are fitted by the thermally activated hopping model $\left[\sigma \propto \exp \left(-T_{0} / T\right)\right]$ at $300-250$ and $210-120 \mathrm{~K}$. The obtained thermal activation barriers $k_{\mathrm{B}} T_{0}$, where $k_{\mathrm{B}}$ is the Boltzmann constant, are 20 and $7 \mathrm{meV}$ for the higher- and lower-temperature ranges, respectively. These are comparable to $12 \mathrm{meV}$ for the surface covered only with the $(4 \times 1)$ phase. The agreement suggests that the domain boundaries also play a role in the present case. Note, however, that the atomically smooth boundary between hex and $(4 \times 1)$ domains as observed by STM [Fig. 2(b)] does not significantly affect electron transport.

The temperature-dependent measurements of LEED and conductivity show that the hex and $(\sqrt{7} \times \sqrt{7})$ phases coexist in a temperature range of $250-210 \mathrm{~K}$ during cooling down. The temperature range is consistent with that reported in a previous STM study for the hex phase without coexisting $(4 \times$ 1) domains [23]. It was suggested [23] that the domains of the hex and $(\sqrt{7} \times \sqrt{7})$ phases have sizes comparable to terrace sizes at intermediate temperatures, which implies that the phase transition proceeds by domain-by-domain conversion. This accounts for the result that the widths of the LEED spots of both phases are almost unchanged during the transition [Fig. 5(a)]. The hysteresis behavior of the $(4 \times 1)$ phase in Fig. 6(a) shows little difference from previous studies for the surface fully covered with the $(4 \times 1)$ phase. The obtained values of $T_{2 \uparrow}$ and $T_{2 \downarrow}$ are in agreement with the previous ones within $1 \mathrm{~K}$. It is therefore concluded that the coexistence with the hex phase does not affect the phase transition of the $(4 \times 1)$ phase.

Finally, we discuss the conductivity drop observed at the transition from hex to $(\sqrt{7} \times \sqrt{7})$. Figure 6(b) shows ARPES Fermi surface maps of the coexisting surface. A part of the circular Fermi contours due to the hex phase is seen in the map measured at RT. For the surface with coexisting $(\sqrt{7} \times \sqrt{7})$ and $(4 \times 1)$ phases at $130 \mathrm{~K}$, the circular Fermi surface disappears completely. In the whole $(1 \times 1)$ SBZ, we were unable to find any Fermi contours except for those of the triple-domain $(4 \times 1)$ phase [18]. This suggests that the conductivity drop at $250-210 \mathrm{~K}$ is due to an electronic MI transition between the hex and $(\sqrt{7} \times \sqrt{7})$ phases.

Hence, the $\sigma-T$ curve below $80 \mathrm{~K}$ is dominated by thermal excitation of carriers across the energy gaps of the $(\sqrt{7} \times \sqrt{7})$ and $(8 \times 2)$ phases. Our previous study of the $(8 \times 2)$ phase showed that the $\sigma-T$ curve is well fitted by $\exp \left(-\Delta / k_{\mathrm{B}} T\right)$ with a constant $\Delta$, where $2 \Delta$ is the band gap of the $(8 \times 2)$ phase $(72 \mathrm{meV})$ [26]. If the energy gap of the $(\sqrt{7} \times \sqrt{7})$ phase is significantly larger than that of the $(8 \times 2)$ phase, the temperature dependence of conductivity remains similar to that of the $(8 \times 2)$ phase. However, fitting to the data of the two-phase coexisting surface gave $2 \Delta=44 \mathrm{meV}$, as shown in Fig. 6(a). This is considerably smaller than that of 
the $(8 \times 2)$ phase, which suggests an even smaller energy gap of the $(\sqrt{7} \times \sqrt{7})$ phase. Metallic band structure was predicted by a previous calculation for the $(\sqrt{7} \times \sqrt{7})$ model of $\theta=10 / 7$ [25]. This does not agree with our ARPES result. The observed hysteresis behavior suggests that the transition is associated with an energy barrier due to large structural transformation. Determination of the atomic structure of the $(\sqrt{7} \times \sqrt{7})$ phase is required in order to establish complete understanding of this complex yet intriguing system.

\section{SUMMARY}

We have studied the atomic structure and phase transition of the dense monolayer phase of In on $\mathrm{Si}(111)$, which was designated as $(\sqrt{7} \times \sqrt{3})$-hex. As a result of LEED and STM experiments, the phase was found to have an incommensurate structure with the In overlayer contracted from $(\sqrt{7} \times \sqrt{3})$ by $2.1 \% \pm 0.3 \%$ in the [ $\overline{1} 10]$ direction. The contraction induces an irregular arrangement of the two types of the protruded atomic chains: zigzag and linear types. First-principles calculations revealed that the total energy of the monolayer phase decreases due to the contraction. Its coverage is revised from 1.4 to 1.43 , which is consistent with $10 / 7$, the nominal coverage of the $(\sqrt{7} \times \sqrt{7})$ phase. The transition from the hex phase to the $(\sqrt{7} \times \sqrt{7})$ phase involves the disappearance of the Fermi surface and the conductivity drop with a large thermal hysteresis, which suggests a first-order MI transition.

\section{ACKNOWLEDGMENT}

The present work was financially supported by JSPS KAKENHI (Grants No. 19H01825, No. 18K03491).
[1] T. Zhang, P. Cheng, W.-J. Li, Y.-J. Sun, G. Wang, X.-G. Zhu, K. He, L. Wang, X. Ma, X. Chen, Y. Wang, Y. Liu, H.-Q. Lin, J.-F. Jia, and Q.-K. Xue, Nat. Phys. 6, 104 (2010).

[2] T. Uchihashi, Supercond. Sci. Technol. 30, 013002 (2017).

[3] C. Brun, T. Cren, and D. Roditchev, Supercond. Sci. Technol. 30, 013003 (2017).

[4] K. Yaji, Y. Ohtsubo, S. Hatta, H. Okuyama, K. Miyamoto, T. Okuda, A. Kimura, H. Namatame, M. Taniguchi, and T. Aruga, Nat. Commun. 1, 17 (2010).

[5] S. Hatta, T. Noma, H. Okuyama, and T. Aruga, Phys. Rev. B 90, 245407 (2014).

[6] K. Yaji, S. Hatta, T. Aruga, and H. Okuyama, Phys. Rev. B 86, 235317 (2012).

[7] S. Stepanovsky, M. Yakes, V. Yeh, M. Hupalo, and M. C. Tringides, Surf. Sci. 600, 1417 (2006).

[8] L. Seehofer, G. Falkenberg, D. Daboul, and R. L. Johnson, Phys. Rev. B 51, 13503 (1995).

[9] J. W. Park and M. H. Kang, Phys. Rev. Lett. 109, 166102 (2012).

[10] K. Uchida and A. Oshiyama, Phys. Rev. B 87, 165433 (2013).

[11] T. Suzuki, J. Lawrence, M. Walker, J. M. Morbec, P. Blowey, K. Yagyu, P. Kratzer, and G. Costantini, Phys. Rev. B 96, 035412 (2017).

[12] T. Shirasawa, S. Yoshizawa, T. Takahashi, and T. Uchihashi, Phys. Rev. B 99, 100502(R) (2019).

[13] E. Rotenberg, H. Koh, K. Rossnagel, H. W. Yeom, J. Schäfer, B. Krenzer, M. P. Rocha, and S. D. Kevan, Phys. Rev. Lett. 91, 246404 (2003).

[14] T. Uchihashi, P. Mishra, M. Aono, and T. Nakayama, Phys. Rev. Lett. 107, 207001 (2011).

[15] M. Yamada, T. Hirahara, and S. Hasegawa, Phys. Rev. Lett. 110, 237001 (2013).
[16] O. Bunk, G. Falkenberg, J. H. Zeysing, L. Lottermoser, R. L. Johnson, M. Nielsen, F. Berg-Rasmussen, J. Baker, and R. Feidenhans'l, Phys. Rev. B 59, 12228 (1999).

[17] H. W. Yeom, S. Takeda, E. Rotenberg, I. Matsuda, K. Horikoshi, J. Schaefer, C. M. Lee, S. D. Kevan, T. Ohta, T. Nagao, and S. Hasegawa, Phys. Rev. Lett. 82, 4898 (1999).

[18] J. R. Ahn, J. H. Byun, H. Koh, E. Rotenberg, S. D. Kevan, and H. W. Yeom, Phys. Rev. Lett. 93, 106401 (2004).

[19] S. Terakawa, S. Hatta, H. Okuyama, and T. Aruga, J. Phys. Condens. Matter 30, 365002 (2018).

[20] S.-i. Park, J. Nogami, and C. F. Quate, J. Microsc. 152, 727 (1988).

[21] J. Kraft, M. G. Ramsey, and F. P. Netzer, Phys. Rev. B 55, 5384 (1997).

[22] D. Shin, J. Woo, Y. Jeon, H. Shim, and G. Lee, J. Korean Phys. Soc. 67, 1192 (2015).

[23] A. A. Saranin, A. V. Zotov, M. Kishida, Y. Murata, S. Honda, M. Katayama, K. Oura, D. V. Gruznev, A. Visikovskiy, and H. Tochihara, Phys. Rev. B 74, 035436 (2006).

[24] A. N. Mihalyuk, A. A. Alekseev, C. R. Hsing, C. M. Wei, D. V. Gruznev, L. V. Bondarenko, A. V. Matetskiy, A. Y. Tupchaya, A. V. Zotov, and A. A. Saranin, Surf. Sci. 649, 14 (2016).

[25] J. W. Park and M. H. Kang, Phys. Rev. Lett. 117, 116102 (2016).

[26] S. Hatta, T. Noma, H. Okuyama, and T. Aruga, Phys. Rev. B 95, 195409 (2017)

[27] M. Yamashita and M. Agu, Jpn. J. Appl. Phys. 23, 1499 (1984).

[28] P. Blaha, K. Schwarz, G. K. H. Madsen, D. Kvasnicka, J. Luitz, R. Laskowski, F. Tran, and L. D. Marks, WIEN2k, an Augmented Plane Wave + Local Orbitals Program for Calculating Crystal Properties (Technische Universität Wien, Vienna, 2018). 